\title{
Application of an internal drainage tube in laparoscopic common bile duct exploration
}

\author{
HANZHANG DONG $^{1}, \mathrm{XI} \mathrm{LIU}^{1,2}$, MINGJIAN LUO ${ }^{1}$, SHAOBIAO KE $^{1}$, JIULIN ZHAN $^{1}$ and ZHIWEI LI ${ }^{1}$ \\ ${ }^{1}$ Department of Hepatobiliary and Pancreatic Surgery, Kanghua Hospital, Dongguan, Guangdong 523080; \\ ${ }^{2}$ Second Clinical Medical College of Guangzhou University of Chinese Medicine, Guangzhou, Guangdong 510006, P.R. China
}

Received July 14, 2021; Accepted September 1, 2021

DOI: $10.3892 / \mathrm{mi} .2021 .14$

\begin{abstract}
Choledocholithiasis is a common disease of the biliary system. The traditional surgical method for this is to remove the gallbladder, open the common bile duct, remove the stones and place a T-tube in the common bile duct for drainage. Common bile duct exploration usually requires a T-tube. Without a T-tube, there is a risk of bile leakage due to pressure in the bile duct. After the T-tube is placed, patients experience some form of discomfort and inconveniences with daily life, and there is also a risk of accidental detachment, as well as a risk of bile leakage when the T-tube is removed. In severe cases, patients may need to be hospitalized again. With advancements being made in surgical instruments and technology, laparoscopic common bile duct exploration has been widely used. Due to the carbon dioxide pneumoperitoneum, laparoscopic common bile duct exploration requires a long period of time for T-tube sinus formation compared with open surgery. Therefore, the extubation time needs to be prolonged in laparoscopic common bile duct exploration. The use of an internal drainage tube may be used in order to avoid the aforementioned disadvantages. Since 2012, the authors have performed laparoscopic common bile duct exploration with the placement of an internal drainage tube for the treatment of common bile duct stones, and have completed $>160$ surgeries. The present study provides a summary of the data of these 160 cases. The 160 patients underwent laparoscopic cholecystectomy. Following the removal of the stones, an internal drainage tube was placed, and the common bile duct incision was primary sutured. All patients were discharged, and there were no complications, such as biliary leakage, biliary bleeding and biliary stricture. On the whole, the present study demonstrates that where possible, the placement of an internal drainage tube in laparoscopic common bile duct exploration
\end{abstract}

Correspondence to: Professor Zhiwei Li, Department of Hepatobiliary and Pancreatic Surgery, Kanghua Hospital, 1000 Dongguan Avenue, Dongguan, Guangdong 523080, P.R. China E-mail: lizhiwei67@126.com

Key words: laparoscopy, biliary stones, common bile duct exploration, internal drainage tube, primary suture is safe and reliable, and may be used to avoid the risk of bile leakage without a T-tube, any inconveniences for patients, and the risk of bile leakage following the removal of the T-tube.

\section{Introduction}

Choledocholithiasis is a common disease of the biliary system requiring hepatobiliary surgery. After the common bile duct is opened and the stones are removed, a T-tube is usually inserted. Without a T-tube, there is a risk of bile leakage due to duodenal papillary sphincter spasm, edema and other reasons following surgery, which is not conducive to the healing of the common bile duct incision. After the T-tube is placed, the patients experience some discomfort and certain inconveniences with daily life, and there is the risk of accidental tube withdrawal. The loss of bile affects the patient's digestive function and is not conducive to post-operative recovery. There is also a risk of bile leakage when the T-tube is removed. An internal drainage tube in the common bile duct may be used to solve these issues. This technique has been studied over the past decade $(1,2)$. Laparoscopic common bile duct exploration has become a routine operation in hospitals in China. The authors have examined $>800$ cases, of which $>160$ cases involved the primary suture of the common bile duct after placing the internal drainage tube. A positive therapeutic effect has been achieved and some problems have arisen. In 2 cases, the internal drainage tube failed to fall out and was removed by duodenoscopy following surgery. There were 31 patients with increased blood amylase levels following surgery, of which 3 cases experienced abdominal pain. The 3 patients were cured after fasting, rehydration and somatostatin treatment. A similar situation appears to exist in endoscopic retrograde cholangiopancreatography (ERCP) surgery (3). In the present study, the authors report their experience with these 160 patients, in the hope that these experiences may prove helpful to other researchers in similar situations.

\section{Patients and methods}

Patient information. The present study was approved by the Ethics Committee of Kanghua Hospital (approval no. 21012). Written informed consent was obtained from the patients. Among the 160 patients, 67 were males and 93 were females, aged between 21 and 76 years. All patients were 
diagnosed with cholecystolithiasis with chronic cholecystitis or acute cholecystitis, and choledocholithiasis by abdominal B-ultrasound, an upper abdominal CT scan and a magnetic resonance cholangiopancreatography examination prior to surgery. There were 142 cases which exhibited an increase in bilirubin levels prior to surgery. The serum total bilirubin level was $82.21 \pm 32.22 \mu \mathrm{mol} / 1$ (normal range, $0-26 \mu \mathrm{mol} / \mathrm{l}$ ) and the serum direct bilirubin level was $57.05 \pm 29.15 \mu \mathrm{mol} / 1$ (normal range, $0-8 \mu \mathrm{mol} / \mathrm{l}$ ) (Table I). All 160 patients underwent a laparoscopic cholecystectomy, laparoscopic choledocholithotomy and common bile duct incision with primary suture following the placement of the internal drainage tube.

Materials and devices used. The internal drainage catheter (Drainage Catheter Locking Pigtail) was obtained from Argon Medical Devices, Inc. (specification, 8F; $2.66 \mathrm{mmx} 25 \mathrm{~cm}$; product model, 755108025). The 5-0 absorbable suture was from Ethicon, Inc. This is a type of fast-absorbing suture composed of polyglactin 910 (product model, W9915). The guide wire was from Terumo Corporation. The diameter of the guide wire was $0.89 \mathrm{~mm}$, the length of the guide wire was $150 \mathrm{~cm}$, and the length of the flexible head was $3 \mathrm{~cm}$ (product model, $\left.\mathrm{RF}^{*} \mathrm{GA} 35153 \mathrm{M}\right)$. The pushing catheter was also from Terumo Corporation. The diameter of the catheter was $1.70 \mathrm{~mm}$ and the length of the catheter was $80 \mathrm{~cm}$ (product model, RF*DG35008M).

Manufacturing method. The tail of the internal drainage catheter was cut off, and the head was kept at a length of $12-13 \mathrm{~cm}$ (with a straight loop head; Fig. 1); a cut was made at $\sim 1 \mathrm{~cm}$ longitudinally at the tail of the retained catheter, and it was sewn with a 5-0 fast-absorbing suture to yield a transverse short arm to create the required internal drainage tube (Fig. 2). The loop head of the internal drainage tube was placed into the duodenum through the duodenal papilla, and the transverse short arm remained in the common bile duct. With the break of the fast-absorbing suture after $\sim 14$ days, the transverse short arm disappeared, the internal drainage tube returned to its original shape, fell into the intestinal tract, and was then discharged from the body. The tip of the head of the push catheter was then cut off, and the head of the push catheter was cut $\sim 3 \mathrm{~mm}$ longitudinally, and stretched into a T-shape to prevent the push catheter from being inserted into the tail of the internal drainage tube during the push process; and to prevent the removal of the internal drainage tube when pulling out the push catheter.

Surgical method. All surgeries were performed under endotracheal general anesthesia. The patients were placed in the reverse Trendelenburg position and tilted to the left. After the pneumoperitoneum was established at a pressure of $12-15 \mathrm{mmHg}$, a standard 4 trocar $30^{\circ}$ laparoscope was introduced to detect lesions. The cystic duct was separated and the distal end of it was clamped with a Weck ${ }^{\circledR}$ Hem-o-lok ${ }^{\circledR}$ Polymer Locking Ligation System (Teleflex Medical, Inc.) to prevent gallstones from falling into the common bile duct. The gallbladder was not removed temporarily. Lifting the gallbladder from the bottom is beneficial to the exposure of the common bile duct. The confluence of the cystic duct and common bile duct was then identified, and the common bile duct was opened with an electrocoagulation hook after confirming that the common bile duct was correct. The stones in the common bile duct were removed using stone removal forceps and the choledochoscope is then placed into the common bile duct for exploration. The unremoved stones were removed again with the stone removal forceps or with the stone removal basket. It was then confirmed that there were no residual stones in the common bile duct.

The guide wire was then introduced into the common bile duct through the choledochoscope, and the head of the guide wire was confirmed to enter the duodenum through the duodenal papilla (Fig. 3). The choledochoscope was then removed and the internal drainage tube was placed along the guide wire (Fig. 4). The push catheter was placed along the guide wire (Fig. 5). The head of the internal drainage tube was then placed into the duodenum, and the transverse short arm was left in the common bile duct. The transverse short arm was located downstream of the mucosal suture of the common bile duct. The correct position of the internal drainage tube was confirmed using a choledochoscope. The gallbladder was then removed. The incision of the common bile duct was then sutured at full-thickness intermittently with a 4-0 absorbable suture, and a drainage tube was placed in the Winslow hole; the surgery was then completed.

\section{Results}

All 160 patients were discharged from the hospital with an average post-operative hospital stay of 4.2 days. At this stage, no post-operative complications, such as bile leakage or biliary stricture were observed. On the morning of the first post-operative day, blood amylase levels were slightly increased in 31 patients. The serum amylase level was 286.17 \pm 64.02 U/1 (normal range, 25-100 U/1) (Table II). Of these patients, 28 patients experienced no abdominal pain and the re-examination of blood amylase levels revealed a return to normal levels a week later. In addition, 3 patients exhibited obvious symptoms of abdominal pain and were treated with somatostatin, and their hospital stay was thus prolonged. These patients were cured and were discharged within 1 week. On the first post-operative day, the abdominal vertical plain film was obtained to determine the position of the internal drainage tube (Fig. 6). All the internal drainage tubes were in the correct position. Among the 160 patients, the internal drainage tube was discharged spontaneously from the body in 158 patients. The fastest draining time was on the 9th day following surgery, and the latest was on the 35 th day. In the majority of patients, the tube was discharged at $\sim 2$ weeks following surgery (Table II). Of note, 1 patient was not discharged on the 55th day and 1 case was discharged on the 63rd day. The two internal drainage tubes of these patients were removed by duodenoscopy following confirmation by abdominal plain film. There was no discomfort during the indwelling of the internal drainage tube. The 2 patients did not experience any discomfort following the removal of the tube.

\section{Discussion}

Cholelithiasis is a common disease. Common bile duct exploration has a history of $>100$ years (4). Bagnato reported laparoscopic common bile duct exploration in 1990 (5). There are advantages and disadvantages of the use of an indwelling T-tube in common 
Table I. Characteristics of the patients in the present study.

\begin{tabular}{lc}
\hline Characteristic & Mean \pm SD or $\mathrm{n}(\%)$ \\
\hline Patients, $\mathrm{n}(\%)$ & 160 \\
Age (years) & $47.12 \pm 11.20$ \\
Sex, $\mathrm{n}(\%)$ & $67(41.88)$ \\
Male & $93(58.13)$ \\
Female & $1.22 \pm 0.26$ \\
Diameter of common bile duct $(\mathrm{cm})$ & $7(4.37)$ \\
Diabetes mellitus, $\mathrm{n}(\%)$ & $142(88.75)$ \\
Increase in bilirubin, $\mathrm{n}(\%)$ & $82.21 \pm 32.22$ \\
Serum total bilirubin $(\mu \mathrm{mol} / \mathrm{l} ;$ & \\
normal range, $0-26 \mu \mathrm{mol} / \mathrm{l})$ & $57.05 \pm 29.15$ \\
Serum direct bilirubin $(\mu \mathrm{mol} / \mathrm{l} ;$ & \\
normal range, $0-8 \mu \mathrm{mol} / \mathrm{l})$ & $131(81.87)$ \\
Abdominal pain, $\mathrm{n}(\%)$ & $37(23.12)$ \\
Fever, $\mathrm{n}(\%)$ & \\
\hline
\end{tabular}

The diameter of the common bile duct is based on pre-operative CT scanning. SD, standard deviation.

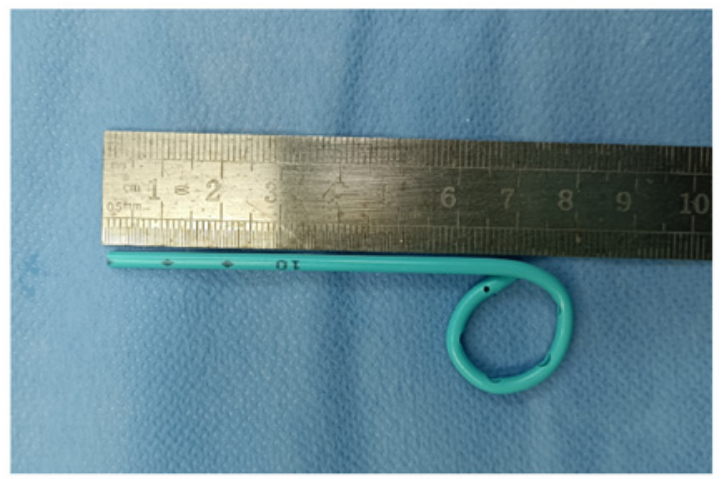

Figure 1. The tail of the internal drainage catheter was cut off and the head was kept at a length of $12-13 \mathrm{~cm}$ (with a straight loop head).

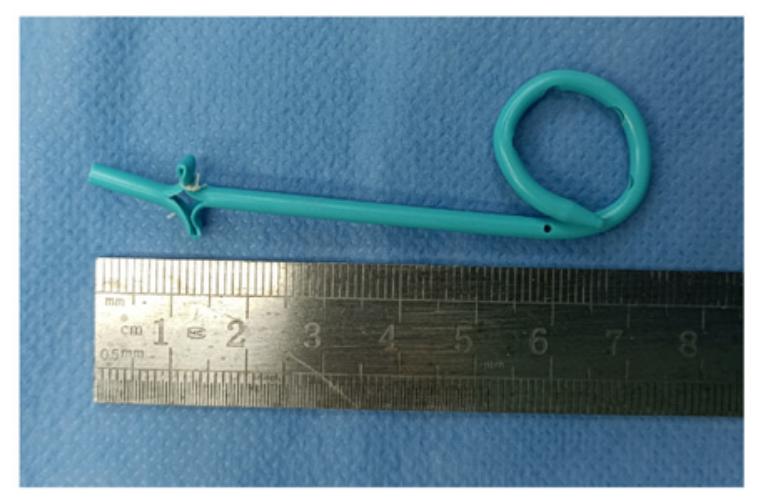

Figure 2 . A cut was made at $\sim 1 \mathrm{~cm}$ longitudinally at the tail of the retained catheter, and it was sewn with a 5-0 fast-absorbing suture to create a transverse short arm.

bile duct exploration. Bile loss can cause dyspepsia, acid-base imbalance and electrolyte disorder. Bile leakage may also occur following the removal of the T-tube. Another surgery is then
Table II. Clinical data of the patients in the present study.

\begin{tabular}{lc}
\hline Variable & Mean \pm SD or $\mathrm{n}(\%)$ \\
\hline Surgery time (min) & $152 \pm 18.33$ \\
Hospital stay (days) & $7.02 \pm 0.35$ \\
Time of abdominal drainage & $2.61 \pm 0.57$ \\
removal (days) & $15.02 \pm 7.61$ \\
Time of internal drainage tube & \\
dislodgement (days) & $31(19.38)$ \\
Increase in blood amylase & \\
following surgery, $\mathrm{n}(\%)$ & $286.17 \pm 64.02$ \\
Serum amylase (U/l; normal & \\
range, 25-100 U/l) & $0(0)$ \\
Biliary leak following surgery, $\mathrm{n}(\%)$ & $0(0)$ \\
Biliary stricture following surgery, $\mathrm{n}(\%)$ & \\
\hline
\end{tabular}

$\mathrm{SD}$, standard deviation.

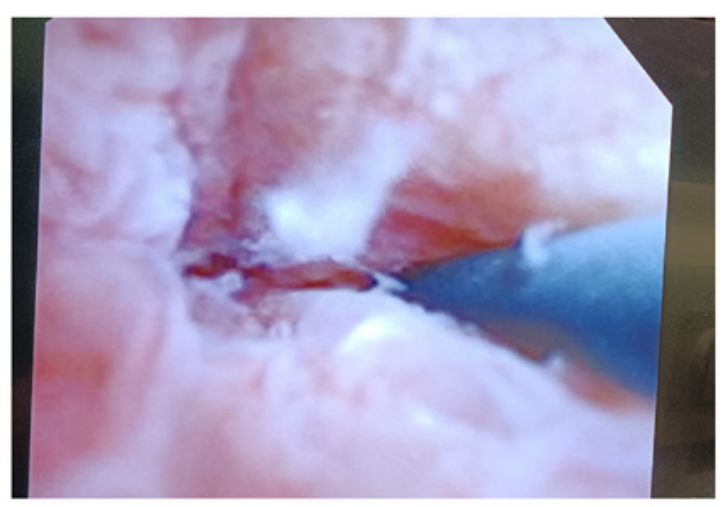

Figure 3. The head of the guide wire was confirmed to enter the duodenum through the duodenal papilla using a choledochoscope.

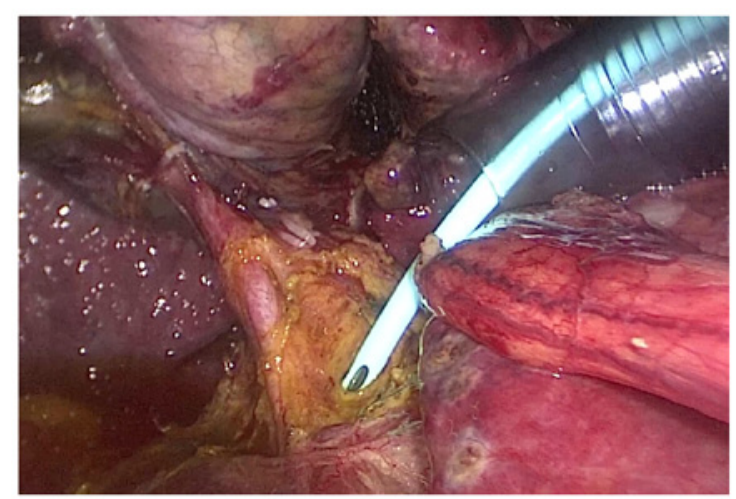

Figure 4. The internal drainage tube is placed along the guide wire.

required in a number of severe cases. In addition, an indwelling T-tube may cause some form of discomfort and inconveniences with daily activities to patients. Compared with open surgery, laparoscopic choledocholithotomy and T-tube drainage required a longer time for the extubation of the T-tube due to the long period of time required for T-tube sinus formation (6). The suture of the common bile duct without a T-tube may cause high 


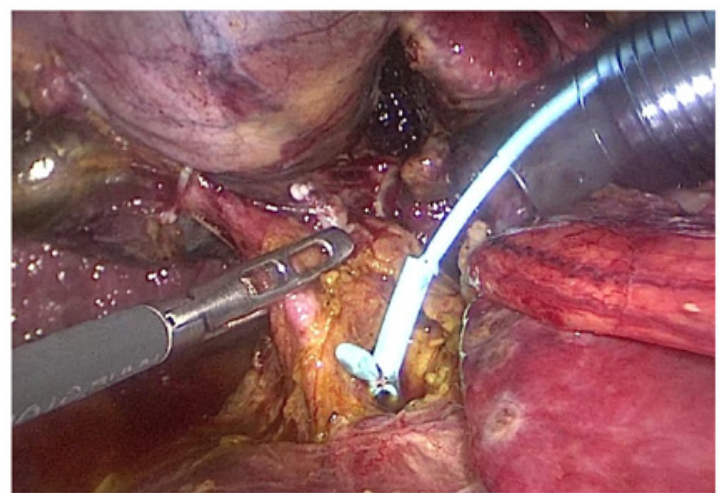

Figure 5. The push catheter is placed along the guide wire.

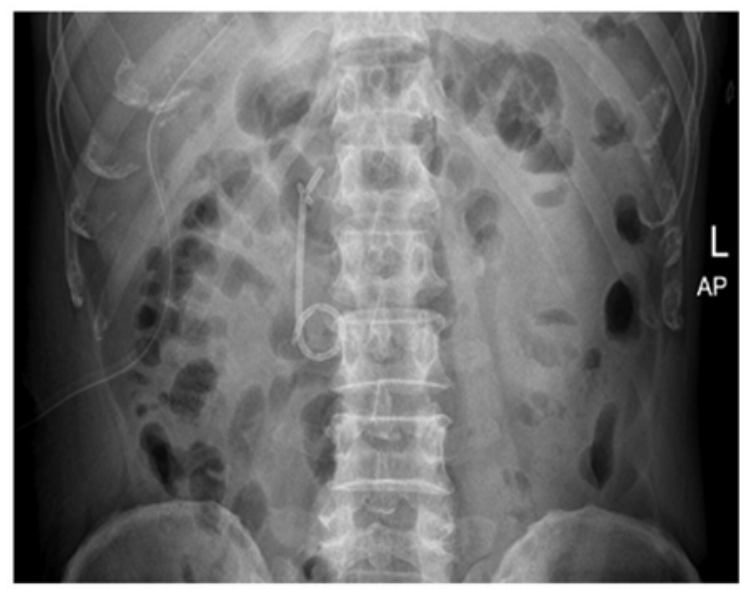

Figure 6. The abdominal vertical plain film was used to determine the position of the internal drainage tube on the first post-operative day. L, left; AP, anterior-posterior position.

pressure in the bile duct due to duodenal papillary sphincter spasm and edema, and the risk of bile leakage is high. The aforementioned issues may be resolved by placing an internal drainage tube instead of a T-tube. As long as the surgical indications are correctly selected, a satisfactory therapeutic effect can be achieved. The surgical indications have reached a consensus at present $(7,8)$. In the authors' experience, the internal drainage tube can be placed in the common bile duct as opposed to the T-tube in the following cases: i) The diameter of the common bile duct is $>0.8 \mathrm{~cm}$; ii) stones are located in the common bile duct, common hepatic duct, or hilar bile duct, which can be removed completely; iii) there is no stricture or new organism in the intrahepatic and extrahepatic bile ducts; and iv) the function of the sphincter of Oddi is normal. It is not recommended to place the internal drainage tube and suture the common bile duct incision in the following situations: i) The stones cannot be removed completely; ii) there are complex intrahepatic and extrahepatic bile duct stones; iii) there is a biliary stricture; and iv) the bile duct wall has severe inflammation, evident congestion and edema, and there are a number of flocculent floating objects on the bile duct wall. It is thus recommended to insert a T-tube $(9,10)$.

There are a number of methods available which can be used to prepare the internal drainage tube and place it in the common bile duct. Some hospitals cooperate with medical device manufacturers to produce special internal drainage tubes and these are then applied in patients. Certain hospitals use an ordinary $16 \mathrm{~F}$ silicone tube to create a barb fitting to prevent the tube falling out, while others use a catheter to prepare an internal drainage tube $(2,11)$. Another important issue is how to place the internal drainage tube safely in a suitable position. The authors' self-made internal drainage tube is a pigtail tube with a diameter of $2.66 \mathrm{~mm}$. The material has proper hardness, good elasticity and multiple side holes. The pigtail end is placed in the duodenum to prevent retrograde entry into the common bile duct. The transverse short arm is placed in the common bile duct to prevent the internal drainage tube from falling out prematurely and entering the intestine. After the fast-absorbing suture is broken down, the transverse short arm disappears by the elastic short arm itself. Generally, the internal drainage tube automatically falls into the intestine and is discharged from the body $\sim 2$ weeks later. In practice, it was found that the internal drainage tube prepared using this method is safe and reliable. The patient's sex and pre-operative bile duct diameter did not affect the time to dislodgement of the internal drainage tube. On the first day following surgery, no abnormality was observed in the abdominal vertical plain film, and all the internal drainage tubes were in the correct position. Of the 160 cases, the internal drainage tubes in 158 cases fell out automatically and were excreted. Of these cases, 1 patient was not discharged at 55 days (the internal drainage tube was removed on day 55 by duodenoscopy) following surgery, and another was discharged at 63 days. In both these cases, the tube was removed using a duodenoscope. When examining the two internal drainage tubes, it was found that the transverse short arms had disappeared, the catheters were unobstructed, and the reasons for not falling out were unknown. Blood amylase levels were slightly increased in 31 patients. A similar situation appears to exist in ERCP surgery. The reasons for this may be duodenal papillary sphincter spasm or edema following surgery. Among these, 3 patients experienced abdominal pain and were treated with somatostatin. They were cured and discharged within 1 week. The internal drainage tube did not cause the patients any discomfort.

In the process of placing the internal drainage tube, the guide wire was placed through the choledochoscope, the internal drainage tube was placed along the guide wire, pushed to the appropriate position with the self-made push catheter, and the position of the internal drainage tube was then confirmed with a choledochoscope. As long there is proficiency with the use of the choledochoscope, the surgeons can place the internal drainage tube in the correct position. In the patients in the present study, no abnormalities were observed in the process of placement. The suture of the common bile duct incision was preferably a full-thickness interrupted suture to maintain the mucosa with a 4-0 absorbable thread. Generally, the needle spacing is $3 \mathrm{~mm}$ and the margin is $2 \mathrm{~mm}$. The tightness of the suture knot should be appropriate, not too tight or too loose. The degree of tightness of the suture knot is without bile leakage. The blood supply of the common bile duct incision will be affected if the suture knot is too tight. Bile leakage can occur if the suture knot is too loose. The drainage tube is placed in the Winslow hole. If the drainage volume fluid is $<10 \mathrm{ml}$ per day and the color of the drainage fluid appears normal (the color of the drainage fluid is dark brown with the presence of bile and red with the presence of blood), the drainage tube can be removed 2 days after the surgery. In 
the case that the patient exhibits fever or abdominal pain, which may indicate biliary leakage or effusion, the extubation can be postponed and an abdominal B-ultrasound examination can be performed to confirm that there is no effusion prior to extubation.

In conclusion, as demonstrated by the present study, as long as the surgical indications are correctly selected, it is safe and reliable to perform internal drainage tube and primary suture the common bile duct incision in laparoscopic common bile duct exploration $(12,13)$. This may be used to avoid the issues associated with the use of a T-tube and the risk of bile leakage without a T-tube. The quality of life of patients is improved and the duration of hospitalization is reduced.

\section{Acknowledgements}

Not applicable.

\section{Funding}

No funding was received.

\section{Availability of data and materials}

The datasets used and/or analyzed during the current study are available from the corresponding author on reasonable request.

\section{Authors' contributions}

$\mathrm{HD}, \mathrm{XL}$ and $\mathrm{ZL}$ were involved in data interpretation, and in the conception and design of the study, and drafted the manuscript. ML, SK and JZ collected the data. ML, SK and JZ confirm the authenticity of the raw data. All authors have read and approved the submitted and final manuscript.

\section{Ethics approval and consent to participate}

The present study was approved by the Ethics Committee of Kanghua Hospital (approval no. 21012). Written informed consent was obtained from the patients.

\section{Patient consent for publication}

The patients or their parents provided consent and agreed for any associated images to be published.

\section{Competing interests}

The authors declare that they have no competing interests.

\section{References}

1. Gurusamy KS and Samraj K: Primary closure versus T-tube drainage after laparoscopic common bile duct stone exploration. Cochrane Database Syst Rev: Jan 24, 200 (Epub ahead of print). doi: 10.1002/14651858.CD005641.pub2.

2. Lyon M, Menon S, Jain A and Kumar H: Use of biliary stent in laparoscopic common bile duct exploration. Surg Endosc 29: 1094-1098, 2015.

3. Andriulli A, Loperfido S, Napolitano G, Niro G, Valvano MR, Spirito F, Pilotto A and Forlano R: Incidence rates of post-ERCP complications: A systematic survey of prospective studies. Am J Gastroenterol 102: 1781-1788, 2007.

4. Verbesey JE and Birkett DH: Common bile duct exploration for choledocholithiasis. Surg Clin North Am 88: 1315-1328, ix, 2008.

5. Bagnato J: Laparoscopic common bile duct exploration. J Miss State Med Assoc 31: 361-362, 1990.

6. Singh AN and Kilambi R: Single-stage laparoscopic common bile duct exploration and cholecystectomy versus two-stage endoscopic stone extraction followed by laparoscopic cholecystectomy for patients with gallbladder stones with common bile duct stones: Systematic review and meta-analysis of randomized trials with trial sequential analysis. Surg Endosc 32: 3763-3776, 2018.

7. Zhan Z, Han H, Zhao D, Song G, Hua J, Xu B and Song Z: Primary closure after laparoscopic common bile duct exploration is feasible for elderly patients: 5 -Year experience at a single institution. Asian J Surg 43: 110-115, 2020.

8. Petelin JB: Laparoscopic common bile duct exploration. Surg Endosc 17: 1705-1715, 2003.

9. Salama AF, Abd Ellatif ME, Abd Elaziz H, Magdy A, Rizk H, Basheer M, Jamal W, Dawoud I and El Nakeeb A: Preliminary experience with laparoscopic common bile duct exploration. BMC Surg 17: 32, 2017.

10. Li KY, Shi CX, Tang KL, Huang JZ and Zhang DL: Advantages of laparoscopic common bile duct exploration in common bile duct stones. Wien Klin Wochenschr 130: 100-104, 2018.

11. Zhen W, Xu-Zhen W, Nan-Tao F, Yong L, Wei-Dong X and Dong-Hui Z: Primary closure versus T-tube drainage following laparoscopic common bile duct exploration in patients with previous biliary surgery. Am Surg 87: 50-55, 2021.

12. Marks B and Al Samaraee A: Laparoscopic Exploration of the common bile duct: A systematic review of the published evidence over the last 10 years. Am Surg 87: 404-418, 2021.

13. Khaled YS, Malde DJ, de Souza C, Kalia A and Ammori BJ: Laparoscopic bile duct exploration via choledochotomy followed by primary duct closure is feasible and safe for the treatment of choledocholithiasis. Surg Endosc 27: 4164-4170, 2013.

This work is licensed under a Creative Commons Attribution-NonCommercial-NoDerivatives 4.0 International (CC BY-NC-ND 4.0) License. 